Journal of Applied Pharmaceutical Science Vol. 7 (07), pp. 062-069, July, 2017

Available online at http://www.japsonline.com

DOI: 10.7324/JAPS.2017.70711

ISSN 2231-3354 (cc)) BY-NC-SA

\title{
Simultaneous quantification of ramipril, glimepiride and metformin in human plasma by ultra-performance liquid chromatography - tandem mass spectrometry
}

\author{
Eman S. Elzanfaly ${ }^{1}$, Sherif A. Abdel-Gawad ${ }^{2 *}$ \\ ${ }^{1}$ Analytical Chemistry Department, Faculty of Pharmacy, Cairo University, Cairo, Egypt. \\ ${ }^{2}$ Pharmaceutical Chemistry Department, College of Pharmacy, Prince Sattam Bin-Abdul Aziz University, Al-Kharj, Kingdom of Saudi Arabia
}

\begin{tabular}{|c|c|}
\hline ARTICLE INFO & ABSTRACT \\
\hline Article history: & \multirow{8}{*}{$\begin{array}{l}\text { Ramipril (RAM), glimepiride (GLM) and metformin (MET) are recently formulated together for the treatment } \\
\text { of diabetes and cardiovascular disorders. This work introduces a liquid chromatography-tandem mass } \\
\text { spectrometric (LC-MS/MS) method for the simultaneous determination of the three drugs in human plasma after } \\
\text { liquid-liquid extraction. This method made use of atorvastatin as internal standard (IS). Analytes were recovered } \\
\text { from plasma by n-hexane: butanol }\left(50: 50 \% \text {, v/v) and subsequently separated on Waters Acquity }{ }^{\mathrm{TM}} \text { UPLC BEH }\right. \\
\text { shield RP-C } \mathrm{C}_{18} \text { column using methanol: water containing } 0.1 \% \text { formic acid }(90: 10 \% \text {, v/v) as a developing } \\
\text { system. The calibration curves were linear }\left(\mathrm{r}^{2}>0.99\right) \text { over a range of } 0.1-1000 \mathrm{ng} / \mathrm{mL} \text { for RAM \& GLM and } \\
250-2000 \mathrm{ng} / \mathrm{mL} \text { for MET. The intra-day and inter-day precisions were below } 14.32 \% \text { and the accuracy was all } \\
\text { within } \pm 15 \% \text {. Moreover, other validation parameters for the proposed method like matrix effect, selectivity, } \\
\text { recovery and stability were adopted. The proposed method can be applied for the sensitive and selective } \\
\text { quantification of the analytes in bioavailability and pharmacokinetics studies. }\end{array}$} \\
\hline $3 / 01 / 2017$ & \\
\hline Ac & \\
\hline Available online: 30 & \\
\hline Key words: & \\
\hline Human plasma; liquid & \\
\hline chromatography; ramipril, & \\
\hline glimepiride, & \\
\hline
\end{tabular}

\section{INTRODUCTION}

Diabetic patients are more prone to heart disease which is the leading cause of premature death in diabetic patients so the concurrent handling of both diseases is an essential issue (Aksnes et al., 2012). Glimepiride (GLM) is an oral sulfonylurea derivative which is indicated for the treatment of type 2 diabetes mellitus (DM). It is 1-[[p-[2-(3-ethyl-4-methyl-2-oxo-3pyrroline-1-carboxamido)ethyl]phenyl]sulfonyl]-3-(trans-4methylcyclohexyl) urea. GLM lowers blood glucose by stimulating the release of insulin from functioning pancreatic

\footnotetext{
* Corresponding Author

Sherif A. Abdel-Gawad, Pharmaceutical Chemistry Department, Collage of Pharmacy, Prince Sattam Bin-Abdul Aziz University, AlKharj, Kingdom of Saudi Arabia; Analytical Chemistry Department, Faculty of Pharmacy, Cairo University, Cairo, Egypt.

Email: sagawad@yahoo.com
}

cells (Massi-Benedetti, 2003). Metformin hydrochloride (MET) is an orally administered biguanide which exerts its effect in lowering of blood glucose level by not only reducing hepatic glucose production and gluconeogenesis but also by enhancing peripheral insulin sensitivity (DeFronzo et al, 1991; Bailey and Turner, 1996). Ramipril (RAM) is an angiotensin converting enzyme (ACE) inhibitor used in treatment of heart failure and hypertension.

RAM is (2S,3aS,6aS)-1-[N-[(S)-1-ethoxycarbonyl-3phenyl-propyl]l-alanyl]perhydrocyclopenta[b]pyrrole-2-carboxylic acid. It is bio-transformed by hepatic esterase into the di-acid ramiprilate, which is the active form (Jackson et al., 2007). The studied drugs are recently formulated in a combined dosage form called Diakit-3 SR (Zydus Cadila Healthcare Ltd, Ahmedabad, India). It is a fixed dose combination of $1 \mathrm{mg}$ GLM, 2.5mg RAM and $500 \mathrm{mg}$ MET. The formula is prescribed for the treatment of type 2 diabetes accompanied by high blood pressure or associated with heart failure. 
Regarding the methods of analysis of the three drugs in biological fluids, several techniques were used for the quantification of RAM in the biological fluids including liquid chromatography-tandem mass spectrometry (LC-MS/MS) (Gupta et al., 2011; Pilli et al., 2011; Patel et al., 2014), high performance liquid chromatography (HPLC) (Kurade et al., 2009) and spectrophotometry (Al-Majed et al., 2001). GLM was determined by LC-MS/MS methods either individually or with some other drugs in biological samples (Salem et al., 2004; Chakradhar et al., 2007; Liu et al., 2010). LC-MS/MS was also used for quantification of MET either alone or in presence of other antidiabetic drugs (AbuRuz et al., 2005; Mistri et al., 2007; Zhang et al., 2007; Marques et al., 2007; Sengupta et al., 2009; Liu and Coleman, 2009). Ultra-performance liquid chromatography (UPLC) has a significant advantage in resoluting the separated anaytes, sensitivity and speed of analysis, especially when coupling with mass spectrometers. It gives better chromatographic separation and narrower chromatographic peak shapes, thereby introducing higher sensitivity with shorter analysis times. Therefore, UPLC-MS is suitable for bioanalytical methods which require high sensitivity and analytical speed (Nakamura., 2011).To date, no reported method was found for the simultaneous determination of RAM, GLM and MET in human plasma. The goal of the present study was to develop and validate a sensitive, simple and selective UPLC-MS/MS method for the simultaneous quantitation of RAM, GLM and MET in human plasma in a single short time run. The proposed method was designed to be able to monitor the plasma concentration of the three drugs from the time of administration till elimination and so the method can be applied in pharmacokinetic studies.

\section{EXPERIMENTAL}

\section{Chemicals}

RAM, GLM and MET were kindly provided by Sanofi Aventis, Cairo, Egypt; their purities were certified to be 99.6, 99.9 and $99.4 \%$, respectively. Atorvastatin (internal standard) was obtained from Aqa international OPTIMUS DRUGS (P) LIMITED, India, its purity was certified to be $99.8 \%$. Human plasma was obtained from VACSERA, Cairo, Egypt. Methanol and formic acid of HPLC grade were supplied by Sigma Aldrich, St. Louis, USA. Water of HPLC grade was supplied by Merck Millipore, Darmstadt, Germany.

\section{LC-MS/MS instrument and conditions}

Waters Acquity ${ }^{\mathrm{TM}}$ (USA) UPLC-system equipped with a quaternary pump, autosampler and coupled with a tandem mass triple quadrupole detector (TQD). Data acquisition and data integration were done using Mass Lynx 4.1 SCN805 Software solutions. Chromatographic separations were performed on Waters Acquity ${ }^{\mathrm{TM}}$ UPLC BEH shield RP-C ${ }_{18}$ column $(150 \mathrm{~mm} x$ $2.1 \mathrm{~mm}, 1.7 \mu \mathrm{m}$ particle size). The mobile phase consisted of methanol: water containing $0.1 \%$ formic acid (90: $10 \%$, v/v). The mobile phase components were degassed before use in a sonicator for 10 minutes. The isocratic chromatographic separation was performed at room temperature at a flow rate of $0.2 \mathrm{~mL} / \mathrm{min}$.

The tandem mass spectrometer was operated using multiple reactions monitoring (MRM). An electrospray ionization (ESI) interface in positive ionization mode was used for all analytes. The suggested mass conditions are presented in Table 1.

\section{Preparation of standard and quality control (QC) samples Stock and working standard solutions}

Standard stock solutions of RAM, GLM and MET $(100 \mu \mathrm{g} / \mathrm{mL})$ and the IS $(100 \mu \mathrm{g} / \mathrm{mL})$ were separately prepared in methanol. Four stock solutions were used separately for the preparation of calibration curve standards and quality control samples. All solutions were stored at $4-8{ }^{\circ} \mathrm{C}$. Preparation of working solutions for calibration and controls was done by appropriate dilutions in methanol. Preparation of IS working solution $(5 \mu \mathrm{g} / \mathrm{mL})$ was carried out by diluting its stock solution with methanol. Calibration working solutions were separately prepared in the range of $1-1000 \mathrm{ng} / \mathrm{mL}$ for RAM \& GLM, and in the range of 250- $2000 \mathrm{ng} / \mathrm{mL}$, for MET, by diluting specific volumes of stock solution with methanol.

\section{Preparation of calibration standards and quality control samples}

Aliquots of $50 \mu \mathrm{L}$ from the working solutions of the three drugs were used to spike $350 \mu \mathrm{L}$ blank plasma thus providing, six plasma standards with the concentrations, $0.1,0.5$, 50, 100, 500 and $1000 \mathrm{ng} / \mathrm{mL}$ (RAM \& GLM) and five plasma standards with the concentrations, 250, 500, 750, 1000 and 2000 $\mathrm{ng} / \mathrm{mL}$ (MET).

Quality control (QC) samples with concentrations, 0.1 $\mathrm{ng} / \mathrm{mL}$ for RAM \& GLM and $250 \mathrm{ng} / \mathrm{mL}$ for MET (lower limit of quantification LLOQ), $0.3 \mathrm{ng} / \mathrm{mL}$ for RAM \& GLM and 750 $\mathrm{ng} / \mathrm{mL}$ for MET (QCL), $400 \mathrm{ng} / \mathrm{mL}$ for RAM \& GLM and 800 $\mathrm{ng} / \mathrm{mL}$ for MET (QCM) and $800 \mathrm{ng} / \mathrm{mL}$ for RAM \& GLM and $1600 \mathrm{ng} / \mathrm{mL}$ for MET (QCH) were used during analysis of validation samples. All samples were mixed using vortex mixer for 1 minute before extraction.

\section{Protocol of extraction}

After spiking the plasma with the three drugs, $50 \mu \mathrm{L}$ of the internal standard working solution was added. The solution was vortexed for 15 seconds then $5 \mathrm{~mL}$ of the extraction mixture (n-hexane: butanol, 50:50\%, v/v) was added. The solution mixture was vortexed for one minute then centrifuged for 5 minutes at $3000 \mathrm{rpm}$. Four millilitres of the organic phase were transferred to centrifuge tubes then evaporated at $60{ }^{\circ} \mathrm{C}$ in a vacuum concentrator then reconstituted by adding $200 \mu \mathrm{L}$ mobile phase. Aliquot of 10 $\mu \mathrm{L}$ of the sample was injected into the chromatographic system. The auto-sampler was kept at $25{ }^{\circ} \mathrm{C}$.

\section{Validation of bioanalytical method}

It was validated according to the United States Food and Drug Administration (FDA) Guidance for Industry Bioanalytical Method Validation (FDA, 2001). 


\section{Selectivity}

Six blank plasma samples were chromatographed before use to identify the contribution of endogenous materials in human plasma which may interfere with RAM, GLM, MET or IS during the preparation of calibration standards and quality control samples.

\section{Linearity and range}

Calibration curves were constructed using, six non-zero samples in the range of $0.1-1000 \mathrm{ng} / \mathrm{mL}$ (RAM \& GLM) and five non-zero samples in the range of 250- $2000 \mathrm{ng} / \mathrm{mL}$ (MET). Additionally, processing of a plasma sample without the IS (a blank sample) was done and a plasma sample with the IS (a zero sample) was also analyzed to ensure the complete interference absence. These samples (blank and zero samples) were not utilized to construct the standard curve. The standard curves were constructed using peak area ratios (analyte to IS) by least-squares linear regression on different days. A calibration curve is accepted when a correlation coefficient (r) of 0.99 or better is obtained.

\section{Lower limit of quantification (LLOQ)}

It was obtained by handling six replicates of plasma samples spiked with the lowest concentration level of the calibration curve (LLOQ).

\section{Precision and accuracy}

They were assessed by analyzing QC samples at the concentrations of the calibration curve. Intra-day precision and accuracy were checked by processing and analyzing six samples of each QC concentration during the same day. Additionally, the inter-day precision and accuracy were evaluated by analyzing six samples of each QC concentration during different days. Percent accuracy was expressed as: [(mean found concentration)/ (nominal concentration) $] \times 100$. Precision was evaluated as $\% \mathrm{CV}$, which should not exceed $15 \%$ except for the LLOQ, where it could reach $20 \%$.

\section{Recovery and matrix effect}

Full details of recovery and matrix effect were discussed by (Matuszewski et al., 1998). Extraction recovery of the three studied drugs was assessed by comparing their peak areas in the pre-extraction samples, which are spiked plasma samples subjected to the whole extraction procedure, with their peak areas in the post extraction samples, which are spiked at the end of the extraction procedure.

Extraction of blank plasma samples from six sources was carried out then spiked with RAM, GLM and MET at QCM level to evaluate the matrix effect. Peak area of RAM, GLM and MET in the post extraction samples (A) where compared to the peak area response of their standard solutions in methanol at equivalent concentrations (B). The ratio (A/B) can be defined as the matrix effect. A value of one indicates no matrix effect, a value more than one suggests ionization enhancement and a fraction value may indicate ionization suppression. Carryover test was carried out to examine any carryover regarding the analyte and the internal standard. The carryover test samples were injected as follows; blank plasma sample, the upper limit of quantification (ULOQ) sample then blank plasma sample.

\section{Analyte stability}

Short term storage stability

Quality control samples were left for a time exceeding the maximum time period expected for routine sample preparation (4 hours) at room temperature. After that time, a calibration curve was freshly constructed with all stability samples in a single run. Comparing of the stability samples with the fresh ones was done at similar concentration.

\section{Stability after preparation}

Quality control samples were prepared and stored in the thermostated auto sampler $\left(25{ }^{\circ} \mathrm{C}\right)$. The prepared samples were analyzed after 12 hours in auto sampler before injection. The standard curves were plotted for all samples in a single run. The stability samples were compared to the fresh ones with the same concentrations.

\section{Freeze and thaw stability}

Evaluation of the three drugs stability in plasma was done by subjecting them to three freeze-thaw cycles of $-85{ }^{\circ} \mathrm{C}$ during 24 hours in three consecutive days. After the third cycle, the samples were analysed and their concentrations were calculated at the same day. Equivalent concentrations from the samples subjected to the cycles and the fresh ones were compared to each other.

\section{Long-term stability}

Freeze storage at $-85{ }^{\circ} \mathrm{C}$ (for one month) was done for three replicates of each QC sample. Calculation of the values was done using a calibration curve prepared at the same day and the mean values for the stored samples were compared with the concentrations of the fresh samples.

Analyte stability requires that the percentage deviation between mean concentrations of the tested stability samples in various conditions and fresh samples' concentrations should be in $\pm 15 \%$ range.

\section{RESULTS AND DISCUSSION}

The aim of this work was to develop a bioanalytical method capable of the simultaneous determination of RAM, GLM and MET that can be able to determine the plasma concentration of the three drugs for pharmacokinetic studies. Due to the inherent selectivity and sensitivity of MS/MS detection, LC-MS/MS was chosen for analysis as it is a useful technique for pharmacokinetic studies because it provides selectivity and sensitivity requirements for bioanalytical methods and enables the determination of drug plasma concentration from the time of administration to the time of elimination. 
Table 1: Main working parameters of the tandem mass spectrometer.

\begin{tabular}{|c|c|c|c|c|}
\hline \multirow[t]{2}{*}{ Parameter } & \multicolumn{4}{|c|}{ Value } \\
\hline & Ramipril & Glimepiride & Metformin & IS \\
\hline Source temperature $\left({ }^{\circ} \mathrm{C}\right)$ & $120^{\circ} \mathrm{C}$ & $120^{\circ} \mathrm{C}$ & $120^{\circ} \mathrm{C}$ & $120^{\circ} \mathrm{C}$ \\
\hline Dwell time per transition (second) & 0.063 & 0.063 & 0.063 & 0.146 \\
\hline Capillary (kV) & $3 \mathrm{kV}$ & $3 \mathrm{kV}$ & $3 \mathrm{kV}$ & $3 \mathrm{kV}$ \\
\hline Desolvation temperature $\left({ }^{\circ} \mathrm{C}\right)$ & $400{ }^{\circ} \mathrm{C}$ & $400{ }^{\circ} \mathrm{C}$ & $400{ }^{\circ} \mathrm{C}$ & $400{ }^{\circ} \mathrm{C}$ \\
\hline Desolvation gas flow (L/Hr) & $800 \mathrm{~L} / \mathrm{hr}$ & $800 \mathrm{~L} / \mathrm{hr}$ & $800 \mathrm{~L} / \mathrm{hr}$ & $800 \mathrm{~L} / \mathrm{hr}$ \\
\hline Cone $(\mathrm{V})$ & 30 & 25 & 20 & 35 \\
\hline Collision energy (V) & 30 & 15 & 15 & 20 \\
\hline Mode of analysis & Positive & Positive & Positive & Positive \\
\hline Ion transition $(\mathrm{Da}) \mathrm{m} / \mathrm{z}$ & $416.97 / 234.19$ & $491.22 / 352.28$ & $129.90 / 60.24$ & $559.39 / 440.27$ \\
\hline
\end{tabular}
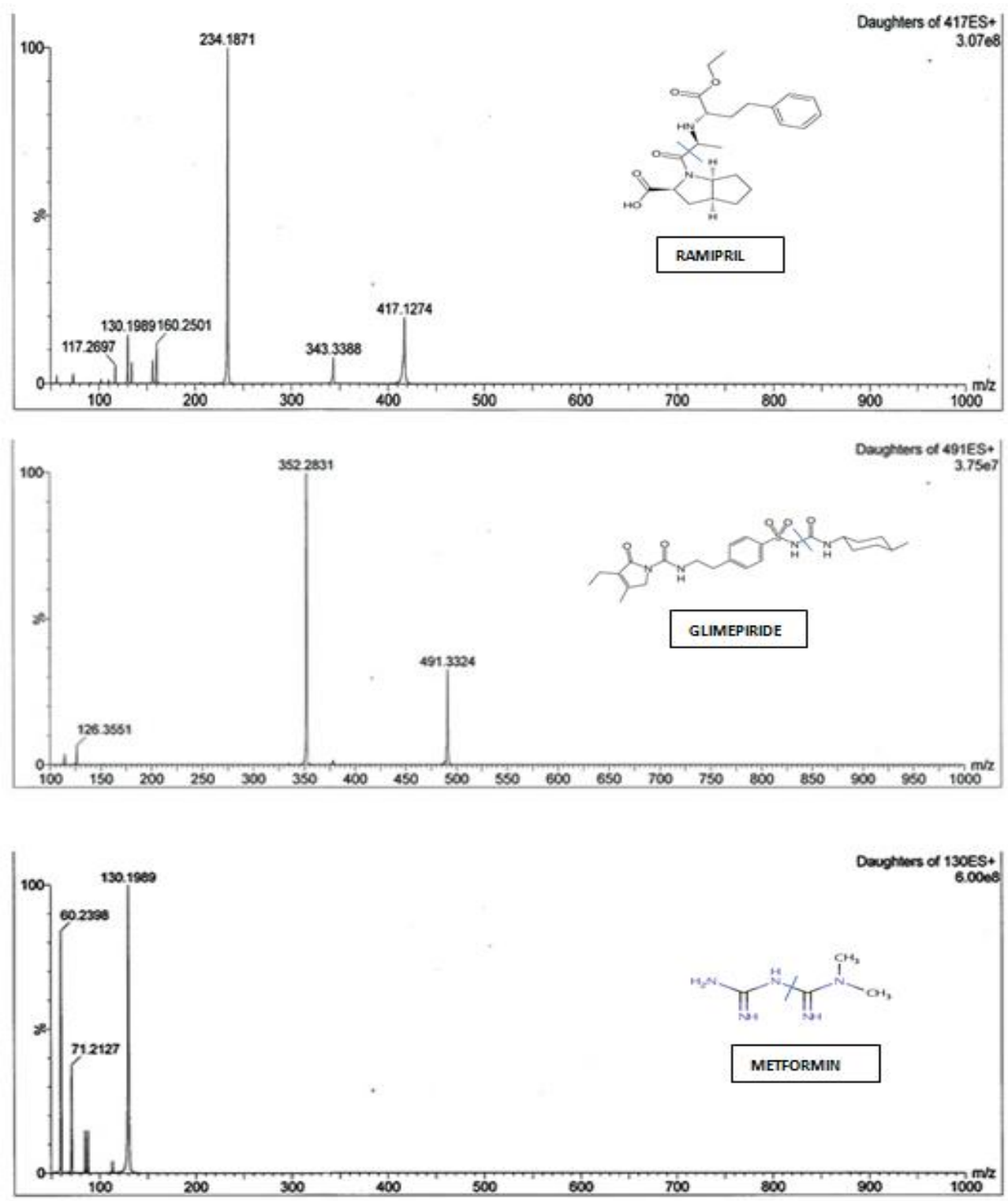

Fig. 1: Mass spectra of the fragmentation products for ramipril, glimepiride and metformin.

\section{Mass spectrometric condition optimization}

The full mass scan of RAM, GLM and MET and IS was obtained in positive ion mode. The $[\mathrm{M}+\mathrm{H}]^{+}$mass spectra of the analytes are presented in Fig. 1. The highest sensitivity of mass transitions was found at $\mathrm{m} / \mathrm{z} 416.97 / 234.19,491.22 / 352.28$,
129.90 / 60.24 and 559.39 / 440.27 for RAM, GLM, MET and IS, respectively. LC-MS/MS in multiple reaction monitoring (MRM) mode introduces the required selectivity and sensitivity for bioanalytical methods. So, the MRM mode was selected for method development. The MRM parameters are given in Table 1. 


\section{Optimization of sample preparation and chromatographic condition}

The procedure of sample preparation should be fast, simple and require minimum amount of reagents with reproducible analyte recovery. In this issue, literature review mentioned several methods for extraction of the studied drugs (Zhu et al., 2002; Tan et al., 2009). In spite of that solid phase extraction technique gives the cleanest samples, yet it is time consuming and requires a lot of optimization steps if compared to liquid-liquid extraction. So, we preferred to use liquid-liquid extraction to decrease processing time and to get the desired analyte recovery. Many extraction solvents like; diethyl ether, dichloromethane, n-hexane, ethyl acetate, butanol and methyl tert-butyl ether were tried to adopt simple and rapid liquid-liquid extraction procedure. Methyl tertbutyl ether was superior for both RAM and GLM but not for MET. Extraction was also tried from different $\mathrm{pH}$ values. MET favors the extraction from basic medium as it will be present in its nonionized form while both RAM and GLM did not give sufficient recoveries from basic medium. The optimum response and recovery for the three drugs were obtained using n-hexane: butanol $(50: 50 \%, \mathrm{v} / \mathrm{v})$ as an extracting solvent from plasma samples without $\mathrm{pH}$ adjustment ( $\mathrm{pH}$ of plasma samples was 6). No interference from any endogenous plasma matrix was noticed. Also, no effect from IS on analyte recovery and/or sensitivity. Although, the used extraction protocol was not the best for MET, but it was excellent for RAM and GLM which should be determined with high sensitivity due to their low dose and hence low plasma concentration levels. It also gave sufficient reproducible recovery that enabled valid determination of MET.

$\mathbf{A}$
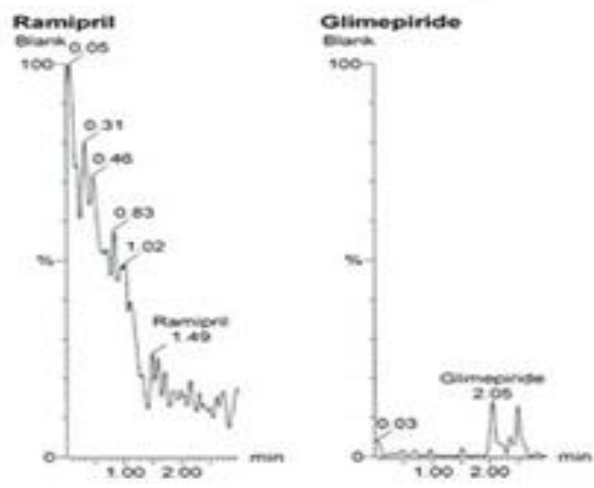

B
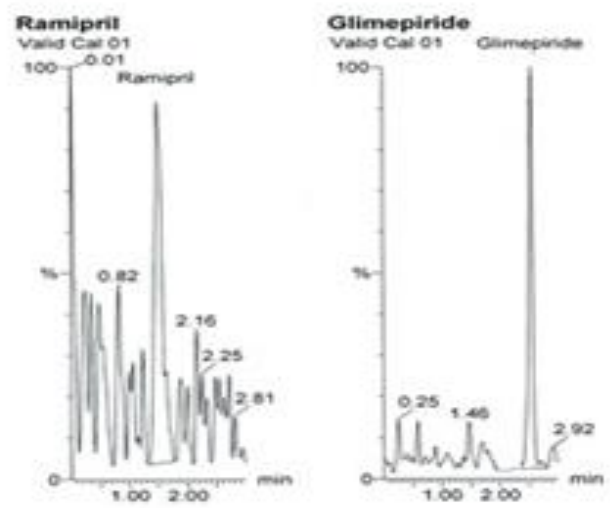

The optimum peak shape, the highest sensitivity for all analytes and IS and also a short chromatographic run time were achieved by optimization of chromatographic conditions. In this work, we tried different columns and various mobile phases such as methanol, water, acetonitrile, formic acid, ammonium acetate and aqueous ammonia. Best resolution was obtained on using Waters Acquity ${ }^{\mathrm{TM}}$ UPLC BEH shield RP-C ${ }_{18}$ column $(150 \mathrm{~mm} \mathrm{x}$ $2.1 \mathrm{~mm}, 1.7 \mu \mathrm{m}$ particle size). The highest sensitivity was achieved with a mobile phase composed of methanol: water containing $0.1 \%$ formic acid (90: $10 \%$, v/v). For choosing the appropriate IS, it is ideal to use isotopically labeled internal standards for all analytes but these are not always commercially available and they are expensive. In this work atorvastatin was used as IS because it was successfully recovered by the extraction procedure and its retention behavior was suitable under the used chromatographic conditions. Under the used chromatographic conditions, a short run time of 3 minutes was obtained with average retention times of 1.6, 1.7, 1.9 and $2.1 \mathrm{~min}$ for MET, RAM, IS and GLM respectively.

\section{Method performance and validation Selectivity}

The high selectivity of LC-MS/MS method is due to its capability of monitoring the fragments derived from the precursor ion of the analytes of interest. Method selectivity was assured by analyzing six blank human plasma extract (Fig.2). No interference from any endogenous substance in the drug-free human plasma was observed at the retention time and MRM channels of the three analytes.
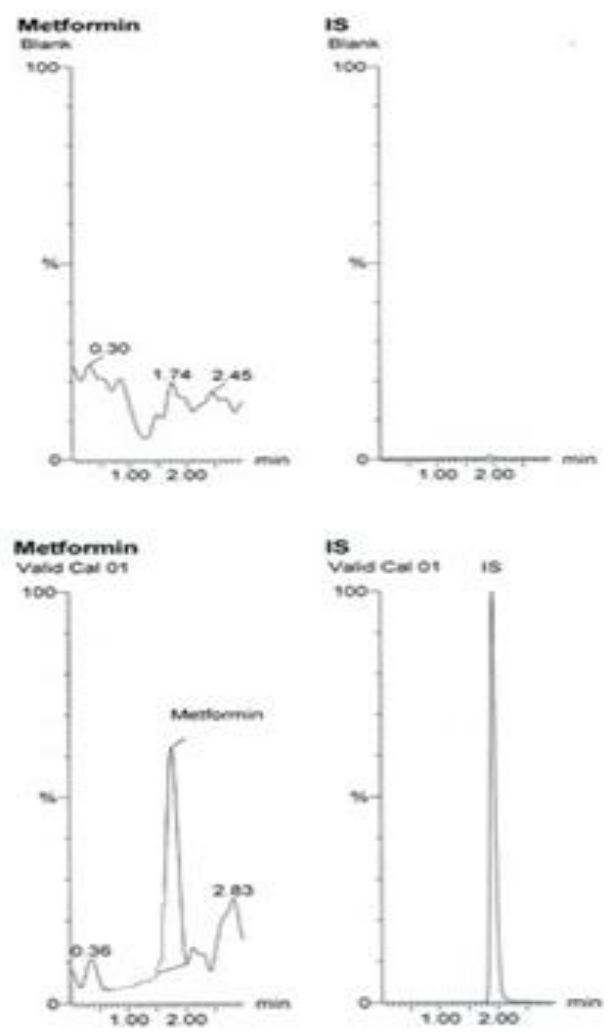

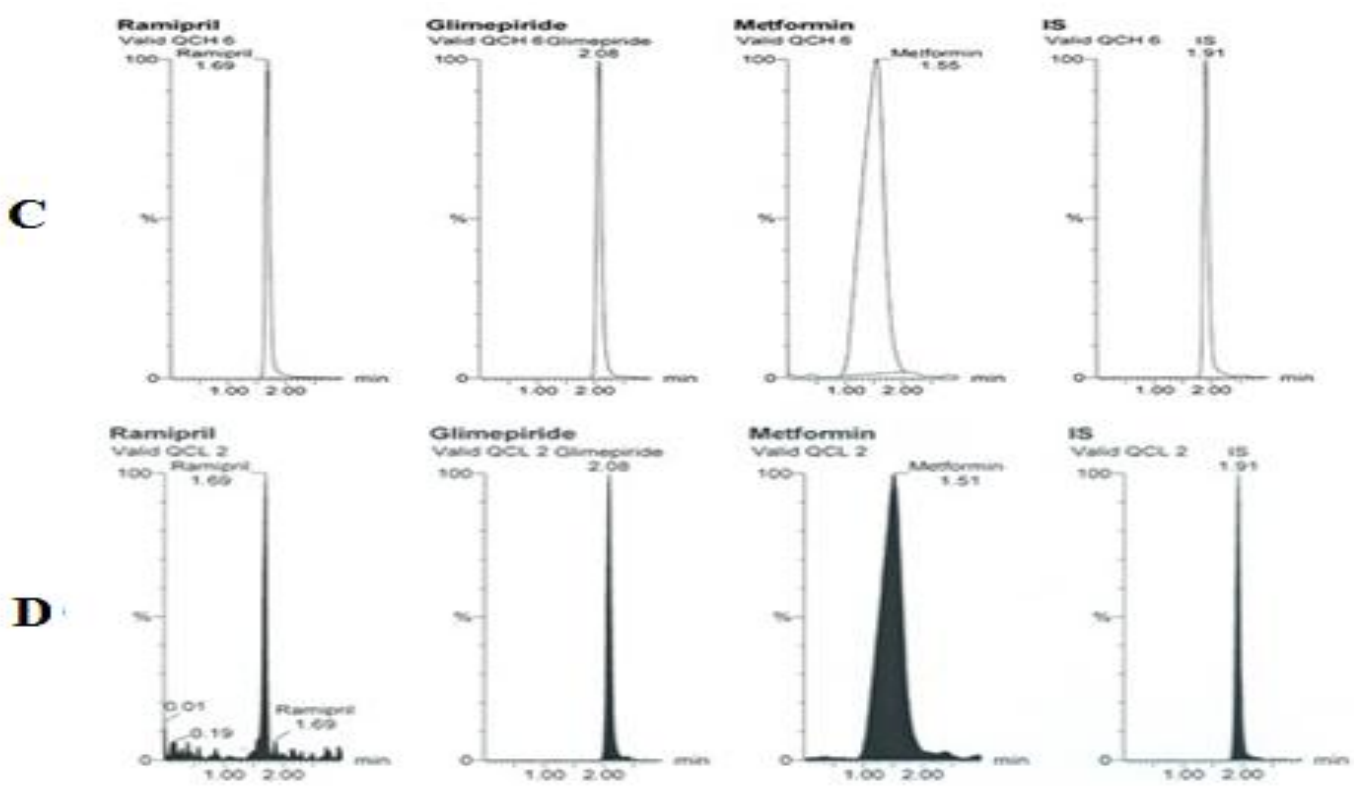

Fig. 2: Representative MRM chromatogram of (A) blank plasma samples, (B) spiked plasma samples at LLOQ with the IS, (C) spiked plasma samples at QCH with the IS and (D) spiked plasma samples at QCL with the IS.

Table 2: Average regression parameters for the calibration curves of ramipril, glimepiride and metformin.

\begin{tabular}{cccc}
\hline & Ramipril & Glimepiride & Metformin \\
\hline Correlation coefficient & 0.9986 & 0.9913 & 0.98955 \\
Slope & 0.0048 & 0.0031 & 0.0002 \\
Intercept & 0.0456 & 0.0548 & 0.0154 \\
\hline
\end{tabular}

Table 3: Precision and accuracy for the determination of ramipril, glimepiride and metformin.

\begin{tabular}{|c|c|c|c|c|c|c|}
\hline & \multicolumn{6}{|c|}{ Recovery Mean recovery \pm RSD \%* } \\
\hline & \multicolumn{3}{|c|}{ Intra-day } & \multicolumn{3}{|c|}{ Inter-day } \\
\hline & Ramipril & Glimepiride & Metformin & Ramipril & Glimepiride & Metformin \\
\hline QCL & $104.24 \pm 13.62$ & $92.19 \pm .8 .22$ & $96.40 \pm .6 .30$ & $89.67 \pm 14.32$ & $108.84 \pm 11.32$ & $97.62 \pm 13.64$ \\
\hline QCM & $97.63 \pm 7.10$ & $95.14 \pm 2.42$ & $92.71 \pm 4.53$ & $92.52 \pm 12.34$ & $105.39 \pm 9.63$ & $97.93 \pm 5.42$ \\
\hline QCH & $98.75 \pm 4.53$ & $101.42 \pm 5.31$ & $95.24 \pm 3.53$ & $98.36 \pm 9.32$ & $93.45 \pm 2.31$ & $102.64 \pm 2.53$ \\
\hline
\end{tabular}

* Mean percentage recovery and RSD\% were calculated using six determinations.

\section{Linearity of calibration curve and lower limit of quantification}

The calibration curves were linear over the concentration range 0.1- $1000 \mathrm{ng} / \mathrm{mL}$ RAM \& GLM (six-point calibration curves, non-zero standards) and 250- $2000 \mathrm{ng} / \mathrm{mL}$ MET (five-point calibration curve, non-zero standards). Calculation of correlation coefficient was done to assure linearity. Table 2 summarizes the calibration curve results.

The LLOQ can be defined as the lowest measurable concentration in the standard curve with acceptable precision and accuracy. It was found to be $0.1 \mathrm{ng} / \mathrm{mL}$, for RAM \& GLM and 250 $\mathrm{ng} / \mathrm{mL}$ for MET, in human plasma with an accuracy of $106.00 \%$, $85.32 \%$ and $90.27 \%$ for RAM, GLM and MET, respectively. Precision (CV\%) was found to be 15.43, 9.87 and $3.67 \%$ for RAM, GLM and MET, respectively. Figure 2 shows the typical chromatograms of LLOQ.

Sufficient sensitivity was observed for a $10-\mu \mathrm{L}$ injection volume with signal to noise ratio of, 28, 381, 82 and 302 for RAM, GLM, MET and IS, respectively, (given by the instrument) at the retention time of the analyte. Under the present LLOQ, RAM and GLM can be determined in human plasma samples containing up to $0.1 \mathrm{ng} / \mathrm{mL}$ while MET can be determined in concentrations starting from $250 \mathrm{ng} / \mathrm{mL}$, as the dose of MET is usually high resulting in a higher plasma concentration than RAM and GLM. These values of LLOQ enables the three drugs to be determined in plasma samples if they are administered together up to their elimination phase after oral administration of a dose of Diakit-3 SR which contain 1mg GLM, 2.5mg RAM and $500 \mathrm{mg}$ MET.

\section{Accuracy and precision measurement}

Table 3 shows the inter- and intra-day precision and accuracy for the analytes in QC samples. The values of inter- and intra-day CV\% were all under $14.32 \%$, which were in the accepted range. With respect to accuracy, all the obtained $\mathrm{CV} \%$ values were in the accepted range $( \pm 15 \%)$.

\section{Recovery and matrix effect}

Recoveries for the three drugs and IS were evaluated as described in the experimental section. The average recoveries of RAM, GLM and MET from spiked plasma samples were 86.19 $\pm 3.42 \%, 96.40 \pm 5.10 \%$ and $70.48 \pm 1.34 \%$ for the quality control 
samples of RAM, GLM and MET, respectively. The recovery of the IS was $84.10 \pm 1.00 \%$ on using concentration of $5 \mu \mathrm{g} / \mathrm{mL}$. Recoveries of the analytes and IS were consistent, precise and reproducible. The low recovery of MET was suggested to be due to its low $\log \mathrm{p}$ value (-0.92) if compared to the other two drugs.

A matrix effect of $0.83,0.89$ and 0.98 was obtained for QCM and hence slight ionization suppression was observed but it had no significant effect on all validation parameters.

\section{Carryover test}

Examination of carryover is required to insure that neither the analytes nor the IS will affect the concentration in the following injections. For the suggested assay no significant carryover was found in blank sample after injection of the ULOQ sample with the working concentration of the IS.

\section{Assessment of stability}

Stability of RAM, GLM and MET after keeping for 4 hours at room temperature was assured. No significant degradation of the three drugs and IS was found after keeping the extracted samples in the auto-sampler at $25^{\circ} \mathrm{C}$ for 12 hours. No difference was observed in the concentrations of QC samples in comparison to the freshly prepared samples after three freeze/thaw cycles. Stability of RAM, GLM and MET under the long term storage conditions $\left(-85^{\circ} \mathrm{C}\right)$ for at least 30 days was also found (Table 4). Stability of stock solutions prepared in methanol was examined at $8^{\circ} \mathrm{C}$ for seven days. The percentage recoveries were 97.09, 100.32, 98.45 and 98.10 for RAM, GLM, MET and IS, respectively, indicating suitable stability during a week. Working solutions were prepared daily just before spiking for both the calibration curve and the QC samples.

Table 4: Stability data of ramipril, glimepiride and metformin under different conditions.

\begin{tabular}{|c|c|c|c|}
\hline \multirow[t]{2}{*}{ Item } & \multicolumn{3}{|c|}{ \% Deviation *from fresh sample concentration } \\
\hline & Ramipril & Glimepiride & Metformin \\
\hline \multicolumn{4}{|c|}{ Short term stability (4h at room temp) } \\
\hline QCL & -0.95 & -7.41 & 0.38 \\
\hline QCM & 1.45 & 9.20 & 14.40 \\
\hline $\mathrm{QCH}$ & 7.94 & -3.00 & -2.30 \\
\hline \multicolumn{4}{|c|}{ Post preparative stability $\left(12 \mathrm{~h}\right.$ at $\left.25^{\circ} \mathrm{C}\right)$} \\
\hline QCL & -3.56 & 9.56 & 4.52 \\
\hline QCM & 4.72 & 2.53 & 7.64 \\
\hline $\mathrm{QCH}$ & 7.65 & 5.73 & 1.53 \\
\hline \multicolumn{4}{|c|}{ Long term stability ( $-20^{\circ} \mathrm{C}$ for 30 days) } \\
\hline QCL & -4.96 & -9.12 & 0.38 \\
\hline QCM & 5.44 & 3.89 & 5.44 \\
\hline $\mathrm{QCH}$ & -4.32 & -9.84 & -4.31 \\
\hline \multicolumn{4}{|c|}{ Freeze- thaw cycles (three cycles) } \\
\hline QCL & -3.31 & -8.56 & 0.38 \\
\hline QCM & 3.92 & 3.89 & 8.07 \\
\hline $\mathrm{QCH}$ & 1.43 & -3.00 & -2.84 \\
\hline
\end{tabular}

$* \%$ deviation $=100 \times($ Stability sample - Fresh sample/ Fresh sample $)$

\section{CONCLUSIONS}

In this work, LC-MS/MS method of high sensitivity and selectivity was adopted and validated for the simultaneous quantification of RAM, GLM and MET in human plasma on using atorvastatin as an internal standard. The proposed method has some advantages including, low sample volume, reproducible recoveries of analytes \& IS with minimum matrix effect, simple sample preparation procedure and capability of simultaneous quantification of all analytes in single short time run. The obtained LLOQ values make the suggested method suitable for monitoring the plasma concentration of the three drugs in plasma for pharmacokinetic studies.

\section{ACKNOWLEDGEMENTS}

The authors gratefully acknowledge CARAS Laboratories (Faculty of Pharmacy, Cairo, Egypt) for providing essential services to carry out this study.

\section{Financial support and sponsorship: Nil.}

Conflict of Interests: There are no conflicts of interest.

\section{REFERENCES}

AbuRuz S, Millership J, McElnay J. The development and validation of liquid chromatography method for the simultaneous determination of metformin and glipizide, gliclazide, glibenclamide or glimperide in plasma. J Chromatogr B, 2005; 817: 277-286.

Aksnes TA, Skårn SN, Kjeldsen SE. Treatment of hypertension in diabetes: what is the best therapeutic option? Expert Rev Cardiovasc Ther, 2012; 10: 727-734.

Al-Majed A, Belal F, Al-Warthan A. Spectrophotometric determination of ramipril (a novel ACE inhibitor) in dosage forms. Spectrosc Lett, 2001; 34: 211-220. $574-579$.

Bailey CJ, Turner RC. Metformin. N Engl J Med, 1996; 334

Chakradhar L, Kallem R, Karthik A, Sundari BT, Ramesh S, Mullangi R, Srinivas NR. A rapid and highly sensitive method for the determination of glimepiride in human plasma by liquid chromatographyelectrospray ionization tandem mass spectrometry: application to a preclinical pharmacokinetic study. J Biomed Chromatogr, 2008; 22: 58-63.

DeFronzo RA, Barzilai N, Simonson DC. Mechanism of metformin action in obese and lean noninsulin-dependent diabetic subjects. J Clin Endocrinol Metab, 1991; 73: 1294-1301.

FDA Guidance for Industry Bioanalytical Method Validation, U.S. Department of health and human services, Food and Drug Administration, Center for Drug Evaluation and Research (CDER), In 2001.

Gupta V, Jain R, Lukram O, Agarwal S, Dwivedi A. Simultaneous determination of ramipril, ramiprilat and telmisartan in human plasma using liquid chromatography tandem mass spectrometry. Talanta, 2011; 83: 709-716.

Jackson E, Brunton LL, Lazo JS, Parker KL. 2007. Goodman \& Gilman's The Pharmacological Basis of Therapeutics. New York, USA: McGraw-Hill.

Kurade V, Pai M, Gude R. RP-HPLC Estimation of Ramipril and Telmisartan in Tablets. Indian J Pharm Sci, 2009; 71: 148-151.

Liu Y, Zhang MQ, Zhu JM, Jia JY, Liu YM, Liu GY, Li S, Weng LP, Yu C. Bioequivalence and pharmacokinetic evaluation of two formulations of glimepiride $2 \mathrm{mg}$ : a single-dose, randomized-sequence, open-label, two-way crossover study in healthy Chinese male volunteers. Clin Ther, 2010; 32: 986-995.

Liu A, Coleman SP. Determination of metformin in human plasma using hydrophilic interaction liquid chromatography-tandem mass spectrometry. J Chromatogr B, 2009; 877: 3695-3700.

Marques MA, Soares AS, Pinto OW, Barroso PT, Pinto DP, Ferreira-Filho M, Werneck-Barroso, E. Simple and rapid method determination for metformin in human plasma using high performance 
liquid chromatography tandem mass spectrometry: application to pharmacokinetic studies. J Chromatogr B, 2007; 852: 308-316.

Massi-Benedetti M. Glimepiride in type 2 diabetes mellitus: a review of the worldwide therapeutic experience. Clin Ther, 2003; 25: 799-816.

Matuszewski BK, Constanzer M L, Chavez-Eng CM. Matrix effect in quantitative LC/MS/MS analyses of biological fluids: a method for determination of finasteride in human plasma at picogram per milliliter concentrations. Anal Chem, 1998; 70: 882-889.

Mistri HN, Jangid AG, Shrivastav PS. Liquid chromatography tandem mass spectrometry method for simultaneous determination of antidiabetic drugs metformin and glyburide in human plasma. J Pharm Biomed Anal, 2007; 45: 97-106.

Nakamura M. Analyses of benzodiazepines and their metabolites in various biological matrices by LC-MS(/MS). Biomed Chromatogr, 2011; 25: 1283-1307.

Patel JR, Pethani TM, Vachhani AN, Sheth NR, Dudhrejiya AV. Development and validation of bioanalytical method for simultaneous estimation of ramipril and hydrochlorothiazide in human plasma using liquid chromatography-tandem mass spectrometry. J Chromatogr B, 2014; 970: 53-59.

Pilli NR, Inamadugu JK, Mullangi R, Karra VK, Vaidya JR, Rao JV. Simultaneous determination of atorvastatin, amlodipine, ramipril and benazepril in human plasma by LC-MS/MS and its application to a human pharmacokinetic study. Biomed Chromatogr, 2011; 25: 439-449.

Salem II, Idrees J, Tamimi JIA. Determination of glimepiride in human plasma by liquid chromatography-electrospray ionization tandem mass spectrometry. J Chromatogr B, 2004; 799: 103-109.
Sengupta P, Bhaumik U, Ghosh A, Sarkar AK, Chatterjee B, Bose A, Pal TK. LC-MS-MS Development and Validation for Simultaneous Quantitation of Metformin, Glimepiride and Pioglitazone in Human Plasma and Its Application to a Bioequivalence Study. Chromatographia, 2009: 69: 1243-1250.

Tan A, Jin W, Deng F, Hussain S, Musuku A, Massé R. Bioanalytical method development and validation using incurred samples-simultaneous quantitation of ramipril and ramiprilat in human EDTA plasma by LC-MS/MS. J Chromatogr B, 2009; 877: 3673-3680.

Zhang L, Tian Y, Zhang Z, Chen Y. Simultaneous determination of metformin and rosiglitazone in human plasma by liquid chromatography/tandem mass spectrometry with electrospray ionization: Application to a pharmacokinetic study. J Chromatogr B, 2007; 854: 9198.

Zhu Z, Vachareau A, Neirinck L. Liquid chromatography-mass spectrometry method for determination of ramipril and its active metabolite ramiprilat in human plasma. J Chromatogr B, 2002; 779: 297 306.

\section{How to cite this article:}

Elzanfaly ES, Abdel-Gawad SA. Simultaneous quantification of ramipril, glimepiride and metformin in human plasma by ultraperformance liquid chromatography - tandem mass spectrometry. J App Pharm Sci, 2017; 7 (07): 062-069. 